Chirurg 2014 · 85:543

DOI 10.1007/s00104-014-2779-x

Online publiziert: 15. Mai 2014

๑) Springer-Verlag Berlin Heidelberg 2014

O. Strobel · M.W. Büchler

Klinik für Allgemein-, Viszeral- und Transplantationschirurgie, Universität Heidelberg

\title{
Dauer und Timing der adjuvanten Chemotherapie nach Resektion eines Pankreaskarzinoms
}

Fluorouracil randomisiert. 985 Patienten, bei denen die Daten zum zeitlichen Ablauf der adjuvanten $\mathrm{CHT}$ ausreichend waren, wurden in die aktuelle Analyse eingeschlossen. 675 (68\%) Patienten erhielten alle 6 Zyklen der adjuvanten CHT, 293 (30\%) Patienten erhielten nur 1 bis $5 \mathrm{Zy}$ klen. Bei 475 (46\%) Patienten wurde die CHT innerhalb von 8 Wochen, bei 528 (54\%) erst 8 bis 12 Wochen postoperativ begonnen. Es gab keine Unterschiede bezüglich der zeitlichen Durchführung der CHT zwischen den Behandlungsgruppen.

\section{Ergebnisse}

schen Überlegungen (Berücksichtigung einer aggressiven Tumorbiologie mit rascher Metastasierung) und auf der Routine der adjuvanten CHT bei anderen soliden Tumoren herrschte bisher die Meinung, dass die adjuvante CHT beim Pankreaskarzinom so rasch wie möglich erfolgen sollte. In den Leitlinien wird ein Beginn der CHT innerhalb 6 Wochen postoperativ empfohlen. Allerdings sind Pankreasresektionen mit einer hohen Morbidität assoziiert und viele Patienten benötigen länger als 6 Wochen, um sich adäquat von der Operation zu erholen. Valle et al. haben den Einfluss der Dauer und des Timings der adjuvanten $\mathrm{CHT}$ auf das Überleben im Patientenkollektiv der European Study Group for Pancreatic Cancer-3 (ESPAC-3-)Studie untersucht.

\section{Methoden}

In der ESPAC-3-Studie wurden $1088 \mathrm{~Pa}$ tienten in die Gruppen Gemcitabine und
Das mediane Gesamtüberleben betrug 23,7 Monate. Lymphknotenbeteiligung, R-Status, Tumordifferenzierung und die Komplettierung der CHT (alle 6 Zyklen) waren unabhängig mit dem Überleben assoziiert. Im Gegensatz dazu war die Dauer bis zum Beginn der CHT im Gesamtkollektiv kein Prognosefaktor. Das mediane Überleben betrug 28,0 Monate bei Komplettierung aller 6 Zyklen vs. 14,6 Monate bei inkompletter CHT (1 bis 5 Zyklen; $\mathrm{p}<0,001)$. Das mediane Überleben betrug 22,6 Monate bei Beginn der CHT innerhalb von 8 Wochen postoperativ vs. 24,2 Monate bei Beginn der CHT mehr als 8 Wochen postoperativ ( $p=0,441)$. Bei Patienten mit inkompletter CHT war ein späterer Beginn der CHT vorteilhaft $(p=0,004)$. Entsprechende Ergebnisse wurden auch bezüglich des rezidivfreien Überlebens beobachtet. Um einen Beobachtungsfehler durch inkomplette Durchführung der CHT bei Patienten mit kur- zer Überlebenszeit auszuschließen, wurden in einer zusätzlichen Analyse alle $\mathrm{Pa}$ tienten ausgeschlossen, die innerhalb der ersten 8 Monate postoperativ verstorben waren. Auch in dieser Analyse bestätigten sich die oben genannten Ergebnisse.

\section{Diskussion}

Die Studie zeigt eindeutig, dass nach Resektion eines Pankreaskarzinoms eine komplette Verabreichung aller 6 Zyklen der adjuvanten $\mathrm{CHT}$ für das Überleben wichtiger ist als ein möglichst früher Beginn der CHT. Ein späterer Beginn der adjuvanten CHT bis zu 12 Wochen postoperativ scheint keinen negativen Einfluss auf das Überleben zu haben. Eine adjuvante CHT kann und sollte also aufgeschoben werden, bis der Zustand der Patienten eine komplette Durchführung aller 6 Zyklen wahrscheinlich macht. Diese Ergebnisse sind angesichts der immer noch hohen Morbidität nach Pankreasresektionen hoch relevant.

\section{Korrespondenzadresse}

\section{PD Dr. O. Strobel}

Klinik für Allgemein-, Viszeralund Transplantationschirurgie, Universität Heidelberg,

Im Neuenheimer Feld 110, 69120 Heidelberg Oliver.Strobel@med.uni-heidelberg.de

Interessenkonflikt. O. Strobel und M.W. Büchler geben an, dass kein Interessenkonflikt besteht. 